\title{
Study on Internal Flow Characteristics of Water Flowmeter using Unsteady Computational Fluid Analysis
}

\author{
Woobin Sim ${ }^{1}$ and Sung-Young Park ${ }^{2}$ \\ ${ }^{1}$ Dept. of Mechanical Engineering, Graduate School, Kongju National Univ., Seobuk- \\ gu, Cheonan-si, Chungcheongnam-do, South Korea \\ ${ }^{2}$ Div. of Automotive \& Mechanical Engineering, Kongju National Univ., Seobuk-gu, \\ Cheonan-si, Chungcheongnam-do, South Korea \\ 1simwbb7@naver.com, ${ }^{2}$ sungyoung@kongju.ac.kr
}

\begin{abstract}
Computational Fluid Dynamics has been carried out for water flowmeter on this study. Two water flowmeters with different geometry have been modeled through the reverse engineering technique. Flow characteristics and rotation per minute of the blade have been calculated using body interaction function between internal passage of the flowmeter and the blade. As results of flow analysis, the flow momentum of FM1 is more evenly applied compared with that of FM2, which means that the blade of FM1 rotates more uniformly than that of FM2. The flow pattern of FM1 is well distributed from the bottom and middle to top, but that of FM2 shows lack of uniformity. It is believed that the uniformity of rotation is disrupted due to the ununiform momentum delivered on the blade wing. If we assume that the rotational speed is linearly proportional to the inlet flowrate, FM1 has 5.9\% and FM2 has $16.8 \%$ error. FM1 is superior to FM2 in linearity. These results could be utilized as basic data to design inner structure of the flowmeter and to develop performance.
\end{abstract}

Keywords: Computational fluid dynamics, Blade, Momentum, Flow characteristics, Water flowmeter

\section{Introduction}

Flow meters are used in a variety of applications in the general industry, petroleum, chemical, shipbuilding, medicine, food, steel, and semiconductors [1]. In particular, various types of flowmeters have been used for the purpose of flow control and commerce in natural gas or water supply pipelines. There are over 100 types of flowmeters in use today. The flowmeter market in Korea is estimated at 120 billion won per year, including domestic water meters and gas meters, and industrial process flowmeters. For reference, the global market for flowmeters is 3.5 trillion won [1][2].

Among the types of flowmeters, there are turbine meters, water meters, or PD meters, such as misaligned gear meters, which measure a rotational speed proportional to the flow rate by inserting a rotor such as an impeller into a flow field [3][4]. All these meters cause pressure losses and increase the uncertainty by reacting sensitively to changes in the flow rate of the fluid entering the meter. Mechanical structures and rotating bodies installed in the fluid flow field may wear or damage over time, changing the flowmeter characteristics, requiring periodic

Article history:

Received (December 2, 2019), Review Result (January 11, 2020), Accepted (February 19, 2020) 
calibration by a flow measurement standard system [3]. [Figure 1] shows an example of a general water meter model used in Korea.

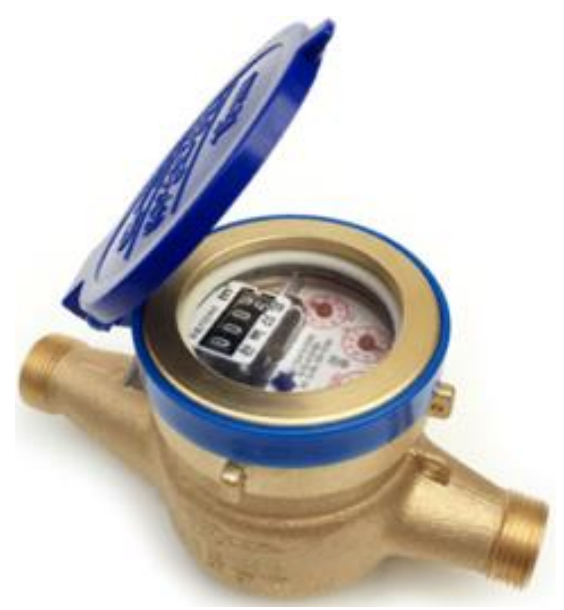

Figure 1. Flowmeter

\section{Main subject}

\subsection{Analysis method}

In this study, the flow analysis of the water meter was conducted using commercial software STAR-CCM +. The models used in the flow analysis were designed using the commercial software CATIA V5. The model in [Figure 2] is named FM1 and the model in [Figure 3] is named FM2 [5].

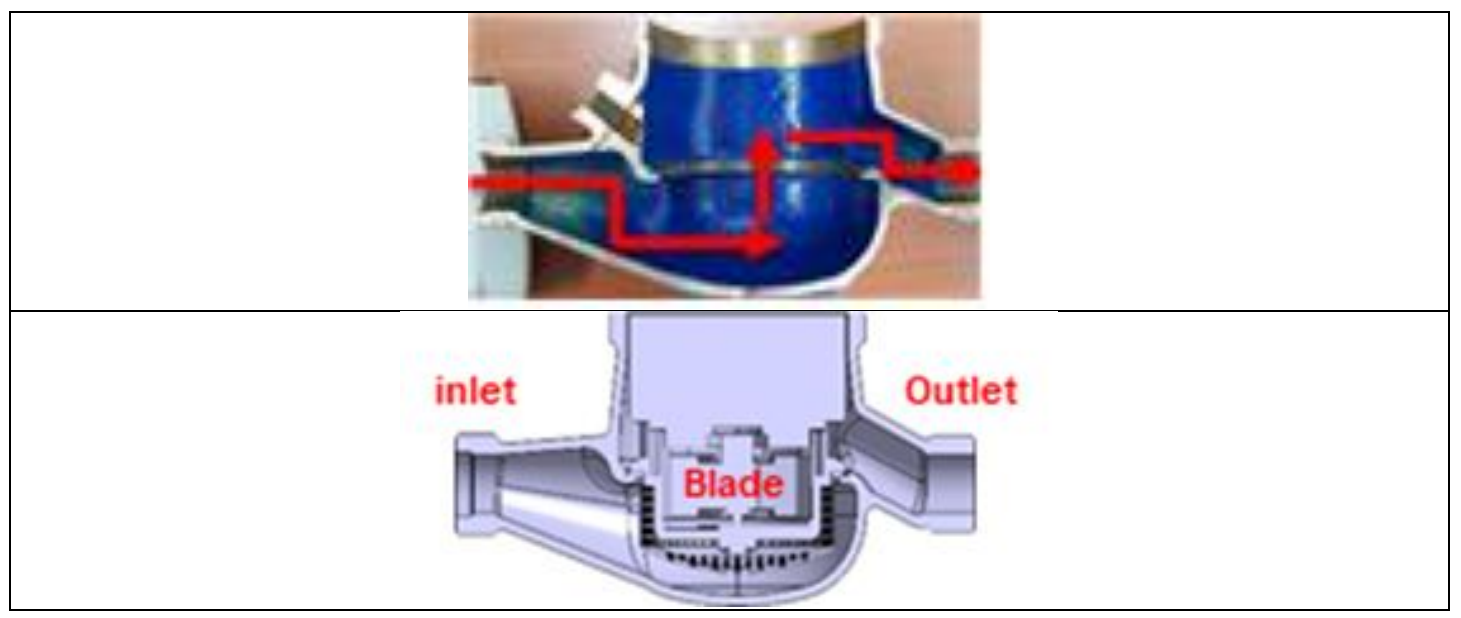

Figure 2. Actual model and 3d modeling of FM1 


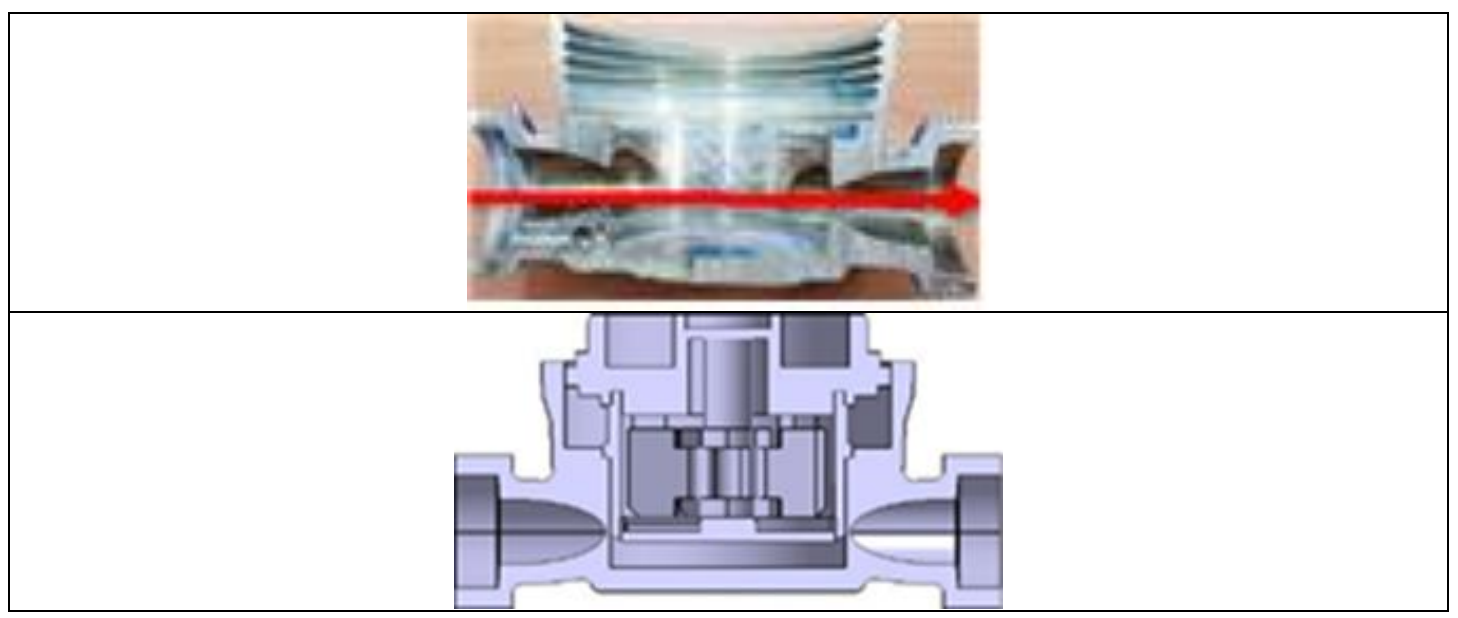

Figure 3. Actual model and 3d modeling of FM2

The flow analysis of the water meter assumes unsteady turbulent flow, and the turbulence model for the flow analysis uses the standard K-Epsilon Turbulence model. The fluid used in the flow analysis was water. In order to simulate the blade rotational movement of the water meter according to the flow of the fluid, the analysis was conducted using the DFBI (Dynamic Fluid Body Interaction) method. The DFBI method can simulate the motion of an object at six degrees of freedom, and the movement of a rigid body against pressure, shear, and other forces exerted by the fluid [6]. This function was used to calculate the rotational speed of the blades, and to calculate the flow trends at each flow rate and the flow velocity distribution around the blades. The computational grid was created using the mesher function in STAR-CCM +, a commercial software. The number of calculation grids was about 2 million FM1 and about 1.67 million FM2. [Figure 4] and [Figure 5] show the flow direction of the FM1 and FM2 and the resulting grid. In the case of FM1, the pressure loss is large when the flow rate entering the inlet flows out to the outlet compared to FM2. FM2 is designed to produce minimal pressure loss on the same shaft as the inlet and outlet, but it cannot guarantee the durability of the product [7].

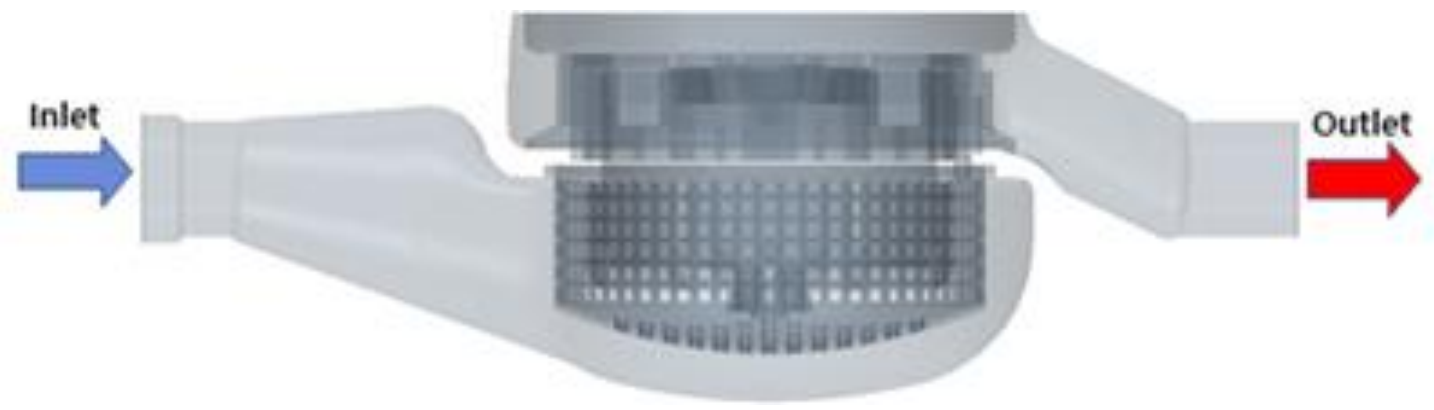

Figure 4. Flow direction and computational grid of FM1 


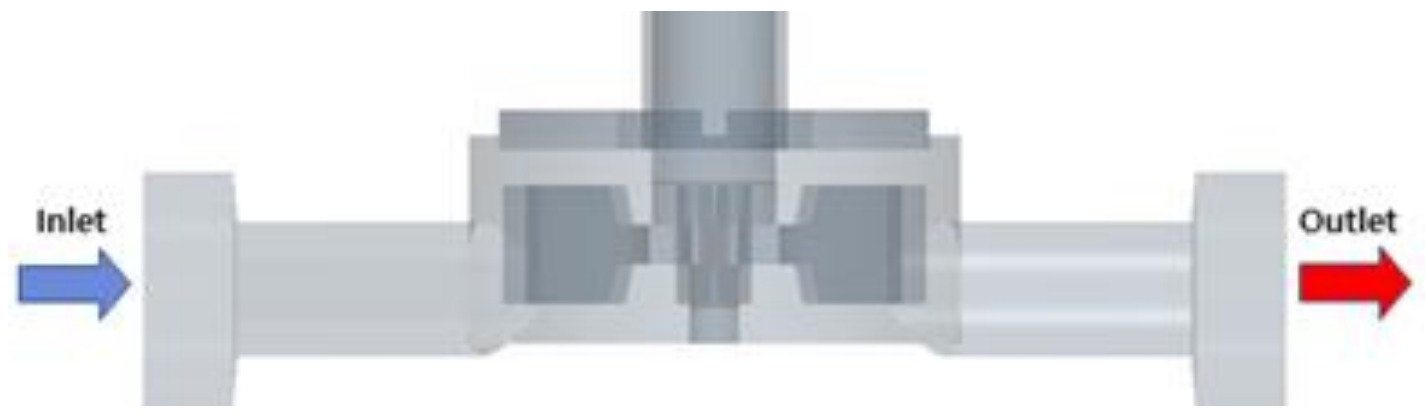

Figure 5. Flow direction and computational grid of FM2

\subsection{Analysis result}

[Figure 6] is the result of the flow analysis inside the flowmeter casing and around the blade when the inlet flow rate is 10lpm. In [Figure 6], FM1 was formed in the upper and lower blades and the corners of the stagnant section during the flow from the inlet to the bottom, resulting in flow resistance. The structure of FM2 is simpler than FM1, but a few side blades receive most of the flow momentum, and the remaining blades act as flow resistance. On the other hand, the blades of the FM1 receive the flow momentum evenly [8].

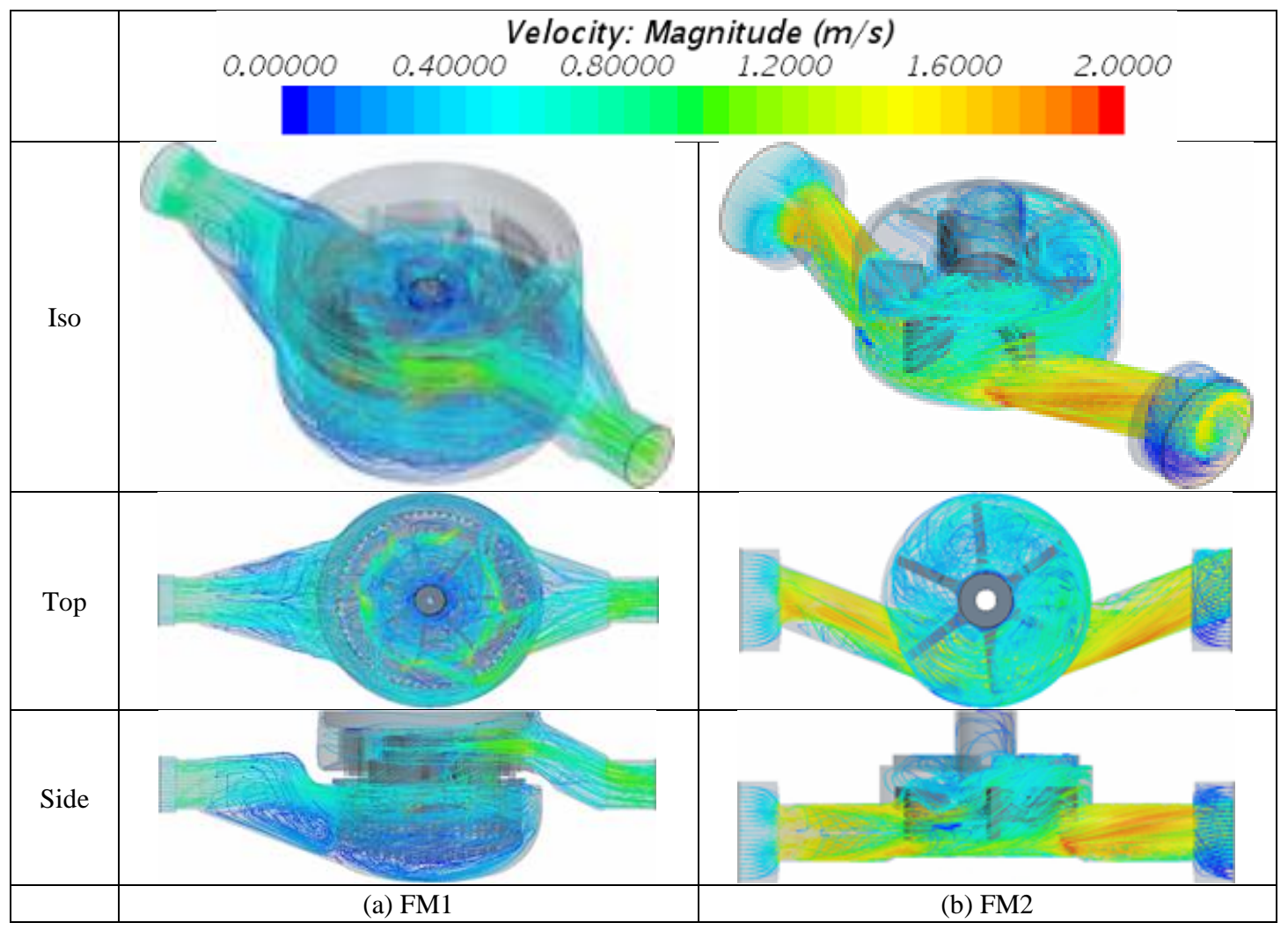

Figure 6. Streamline and velocity @ inlet flow=201pm

[Figure 7] is the flow analysis result when the inlet flow rate is $20 \mathrm{lpm}$. As shown in [Figure 7], there is a clear difference in the flow rates between the models, and the flow of the FM1 is evenly generated on the blade surface compared to the FM2. The flow momentum of FM1 
causes the rotation of the blade well. The FM2 is supplied with momentum of flow only to some of the blades, with the inlet and outlet inclined about $15^{\circ}$ to the tangential surface. In the case of this flow, dead volume is generated in the conduit portion of the blade inlet and outlet. This phenomenon is expected to increase as the flow velocity increases, which may act as an obstacle to precise flow rate measurement at high flow rates.

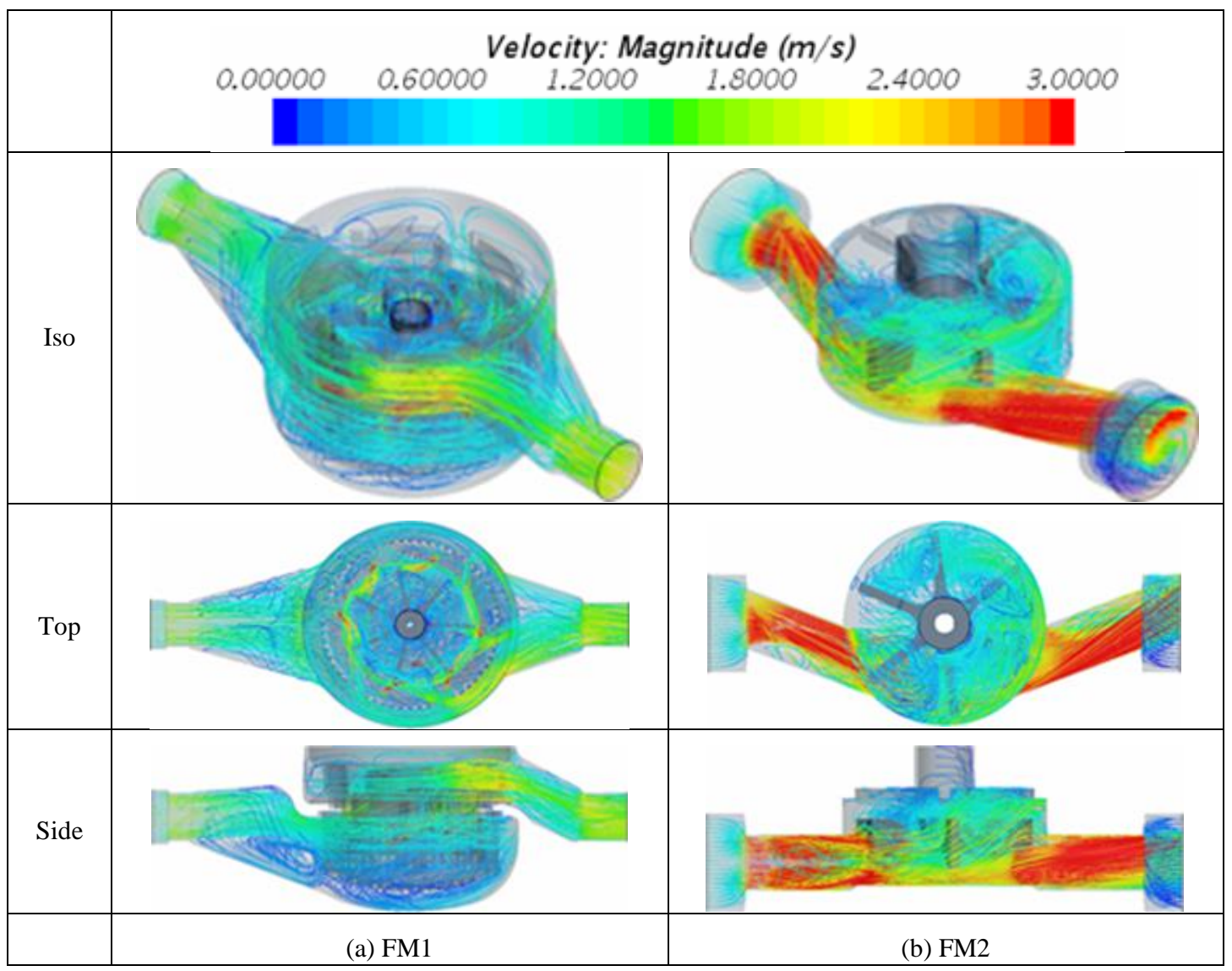

Figure 7. Streamline and velocity @ inlet flow=201pm

\section{Conclusion}

In this study, the flow characteristics, blade rotation speed, and pressure drop for water meters with different structures were calculated through the flow analysis, and the following conclusions were drawn.

1. Two flowmeter models with different shape structures were modeled using reverse engineering, and the flow characteristics and blade rotation speed were calculated by applying the DFBI function to the inside of the flowmeter and the blades.

2. As a result of considering the internal flow characteristics, the flow rate of FM1 was evenly applied to the blade surface compared to FM2, and the flow momentum of FM1 generated the blade rotation well. In the FM2, the momentum of the flow is supplied only to a part of the blade, and the inlet and the outlet are inclined about $15^{\circ}$ to the tangential surface, and dead volume is generated in the pipeline part of the blade inlet and the outlet. The dead volume is expected to act as a factor that inhibits precise flow measurement at high flow rate. 
Through this study, the flow characteristics of the two models with different shape structures were identified and the rotational speeds were calculated. The results of this study could be supplemented through future experimental study and could be used as basic technical data for improving the accuracy of the flowmeter.

\section{Acknowledgements}

This work was supported by the Technological Innovation R\&D Program (G21S269976501) funded by the Small and Medium Business Administration (SMBA, Korea)

\section{References}

[1] Baek Jong-seung, "Commentary flowmeter technology trends," The KSFM Journal of Fluid Machinery, vol.3, no.1, pp.74-78, (2000)

[2] Jin Chul Joo, Ho Sang Ahn, Chang Hyuk Ahn, Kyung Rok Ko, and Hyun Je Oh, "Recent developments and field application of foreign waterworks automatic meter reading," Journal of Korean Society of Environmental Engineers, vol.34, no.12, pp.863-870, (2012) DOI: https://doi.org/10.4491/ksee.2012.34.12.863

[3] Do-Hyung Lee, Hyun-Koo Kim, and Tae-Suk Shin, "R \& D trend of flowmeters," The KSFM Journal of Fluid Machinery, vol.11, no.1, pp.95-99, (2008)

[4] W. Jaewoo Shim, "Measurement of two-phase flow using turbine flowmeter," Journal of Ocean Engineering and Technology, vol.12, no.2, pp.147-152, (1998)

[5] In-Hwan Yeo, Jong-Hoo Paik, Young-Jin Lee, Min-Chul Shin, and Jae-Woo Park, "Optimization and efficiency improvement of flow meter blade using computational fluid dynamics," Korean Geo-Environmental Society, (2011)

[6] CD-Adapco, Star-CCM+ver. 12.04, "User guide," (2017)

[7] Jae Yun Kim, Jung-Hui Ji, Young Sub Lee, and In-Ha Sung, "Design and analysis of flow channel in a pipe with respect to flow rate for measurement reliability of a watermeter," Korean Tribology Society, (2014)

[8] W.H. Kim, Y.J. Lee, J.S. Yang, Y.B. Kim, and B.S. Kim, "Numerical study on the improvement of venturi flowmeter with fouling effect," Journal of Computational Fluids Engineering, vol.21, no.2, pp.40-46, (2016) DOI: https://doi.org/10.6112/kscfe.2016.21.2.040 